\title{
Proteome analysis of the dystrophin-deficient MDX diaphragm reveals a drastic increase in the heat shock protein cvHSP
}

\author{
Philip Doran ${ }^{1}$, Geraldine Martin ${ }^{1}$, Paul Dowling ${ }^{2}$, Harald Jockusch ${ }^{3}$ and Kay Ohlendieck ${ }^{1}$ \\ ${ }^{1}$ Department of Biology, National University of Ireland, Maynooth, Co. Kildare, Ireland \\ ${ }^{2}$ School of Biotechnology, Dublin City University, Dublin, Ireland \\ ${ }^{3}$ Faculty of Biology, University of Bielefeld, Bielefeld, Germany
}

Duchenne muscular dystrophy is the most commonly inherited neuromuscular disorder in humans. Although the primary genetic deficiency of dystrophin in X-linked muscular dystrophy is established, it is not well-known how pathophysiological events trigger the actual fibre degeneration. We have therefore performed a DIGE analysis of normal diaphragm muscle versus the severely affected x-linked muscular dystrophy (MDX) diaphragm, which represents an established animal model of dystrophinopathy. Out of 2398 detectable 2-D protein spots, 35 proteins showed a drastic differential expression pattern, with 21 proteins being decreased, including Fbxo11-protein, adenylate kinase, $\beta$-haemoglobin and dihydrolipoamide dehydrogenase, and 14 proteins being increased, including cvHSP, aldehyde reductase, desmin, vimentin, chaperonin, cardiac and muscle myosin heavy chain. This suggests that lack of sarcolemmal integrity triggers a generally perturbed protein expression pattern in dystrophin-deficient fibres. However, the most significant finding was the dramatic increase in the small heat shock protein cvHSP, which was confirmed by 2-D immunoblotting. Confocal fluorescence microscopy revealed elevated levels of cvHSP in MDX fibres. An immunoblotting survey of other key heat shock proteins showed a differential expression pattern in MDX diaphragm. Stress response appears to be an important cellular mechanism in dystrophic muscle and may be exploitable as a new approach to counteract muscle degeneration.

\section{Keywords:}

Cardiovascular heat shock protein / DIGE / MDX diaphragm / Muscular dystrophy / Skeletal muscle proteomics

\section{Introduction}

Individual skeletal muscle proteins are relatively difficult to isolate and analyse biochemically due to the rigid structure of muscle fibres and the high density of contractile proteins and

Correspondence: Professor Kay Ohlendieck, Department of Biology, National University of Ireland, Maynooth, Co. Kildare, Ireland

E-mail: kay.ohlendieck@nuim.ie

Fax: $+353-1-708-3845$

Abbreviations: BVA, biological variation analysis module; DIA, differential in-gel analysis; MDX, x-linked muscular dystrophy; smHSP, small heat shock protein
Received: February 1, 2006

Revised: April 26, 2006

Accepted: April 26, 2006 intracellular membrane systems. Subcellular fractionation procedures are often hampered by the cross-contamination with highly abundant sarcoplasmic reticulum vesicles, and high background levels of myosin, actin, troponin and tropomyosin can make the purification of low-abundance muscle proteins extremely difficult [1]. However, because of the high frequency of genetic muscle disorders there is considerable interest in studying protein expression and protein-protein interactions in normal versus diseased skeletal muscles [2]. Proteomic screening of total cellular extracts [3] embodies the ideal tool to overcome some of the technical problems of basic myology [4]. Although 2-D gels of fibre homogenates do not represent the entire muscle protein complement, optimised electrophoretic procedures can 
separate a large and representative proportion of key metabolic, structural, regulatory and contractile elements [5]. If one accepts that certain high-molecular mass proteins, very low-density components and proteins with extreme $\mathrm{p} I$ values or certain PTMs may be under-represented in 2-D gels [6], DIGE is one of the most powerful tools for conducting comparative biochemical investigations [7-9]. Unlu et al. [7] first described this proteomics technology, and the first published evaluation of its 2D software analysis was by Tonge et al. [8]. Advanced DIGE, using an internal pooled standard, is a highly accurate quantitative method that enables multiple protein samples to be separated on the same 2-D gel, thereby greatly reducing the introduction of potential artefacts due to gel-to-gel variations [9].

Comparative proteomics approaches are well suited for studying the molecular pathogenesis of inherited muscular disorders [10]. Often the identification of the primary genetic abnormality and the elucidation of secondary changes may not lead to a comprehensive understanding of the cellular downstream events that render muscle fibres more susceptible to necrosis [11]. In conjunction with findings from gene expression profiling studies, proteomics can be used to study how a specific mutation can perturb the global protein expression pattern in a muscle. From the proteomics-based categorisation of proteins with a drastically changed expression, conclusions about the compensatory up-regulation or degeneration-dependent loss of specific cellular components can be made. In the case of Duchenne muscular dystrophy, the most commonly inherited neuromuscular disease in humans [12], the primary deficiency in the membrane cytoskeletal protein dystrophin has been established for nearly two decades [13]. The loss of dystrophin triggers the secondary loss of various surface proteins, such as dystroglycans, sarcoglycans, syntrophins, dystrobrevin and sarcospan [14], as well as associated signalling elements including the neuronal isoform of nitric oxide synthase [15]. The molecular collapse of the dystrophin-glycoprotein complex is believed to weaken the linkage between the extracellular matrix and the actin membrane cytoskeleton in muscle fibres [16]. However, it is not well understood how these primary and secondary changes in fibre integrity finally lead to disturbances of signal transduction, energy metabolism, ion homeostasis and excitation-contraction coupling [11]. To address this question, we have used DIGE analysis of the $\mathrm{x}$ linked muscular dystrophy (MDX) diaphragm to study potential global disturbances in the protein expression profile of dystrophin-deficient fibres. The MDX mouse diaphragm model of Duchenne muscular dystrophy exhibits severe fibre degeneration [17] making it a suitable cellular system to study the effects of dystrophin deficiency [18].

Large-scale transcriptomics and proteomics studies have recently been performed to study normal skeletal muscle and muscle disease processes. Skeletal muscle proteomics has been successfully applied to evaluate differential expression levels in fast versus slow fibres [19], chronic low-frequencystimulated fast muscle [20], the identification of age-de- pendent protein nitration [21], the analysis of O-linked $\mathrm{N}$-acetylglucosamine proteins [22], the expression profiling during muscle growth [23], and the general cataloguing of muscle protein species $[5,6]$. Besides comparative transcriptomics screening of dystrophin-deficient muscle specimens [24-28], MS-based proteomics led to the identification of novel biomarkers in MDX tissues [29], such as adenylate kinase [30], calsequestrin [31] and regucalcin [32]. These investigations were performed with conventional nonfluorescence gel electrophoresis techniques. In contrast, here we have applied a several-fold more sensitive proteomics method, DIGE [9], and have determined the expression levels of fluorescently tagged normal versus dystrophic diaphragm proteins.

\section{Materials and methods}

\subsection{Materials}

CyDye DIGE fluor minimal dyes Cy2, Cy3 and Cy5, electrophoresis grade chemicals, IPG strips of $\mathrm{pH}$ 3-10 (linear) and IPG buffer of $\mathrm{pH}$ 3-10 for IEF, the 2-D Quant kit for determination of protein concentration in electrophoretic samples, the 2-D Clean-Up kit for removal of contaminants prior to IEF, and ACN were obtained from Amersham Biosciences/GE Healthcare (Little Chalfont, Bucks., UK). DNase-I enzyme, ultrapure lysine for quenching the DIGE labelling reaction, colloidal CBB concentrate and the presilconisation medium Sigmacote were from Sigma Chemical Company (Dorset, UK). For desalting of MS samples, C-18 ZipTips were purchased from Millipore Ireland B.V. (Carrigtwohill, Cork, Ireland). A matrix kit containing CHCA was obtained from Laserbiolabs, SophiaAntipolis, France. For peptide generation, sequencing-grade modified trypsin was purchased from Promega (Madison, WI, USA). Ultrapure Protogel acrylamide stock solutions were purchased from National Diagnostics (Atlanta, GA, USA). Primary antibodies were from Abcam, Cambridge, UK (pAb ab21128 to cvHSP; pAb ab23855 to GRP75), StressGen Bioreagents, Victoria, Canada (pAb SPA-796 to HSP20; pAb SPA801 to HSP25; mAb LK-2 to HSP60; mAb BB70 to HSP70; mAb 16F1 to HSP90; pAb SPA-1103 to HSP110) and Visionbiosystems Novocastra, Newcastle upon Tyne, UK (mAb 8D5 to $\beta$-dystroglycan). Peroxidase-conjugated secondary antibodies were purchased from Chemicon International (Temecula, CA, USA) and Alexa Fluor-conjugated secondary antibodies were from Invitrogen Molecular Probes (Bio Sciences, Dun Laoghaire, Ireland). The DNA-binding dye diamidinophenyindole was from Sigma. Chemiluminescence substrates, Immobilon NC-pure NC membranes and protease inhibitors were obtained from Pierce and Warriner (Chester, UK), Millipore (Bedford, MA, USA), and Roche Diagnostics (Mannheim, Germany), respectively. Superfrost Plus positively charged microscope slides were from Menzel Glässer (Braunschweig, Germany). All other chemicals used were of analytical grade and purchased from Sigma Chemical Company. 


\subsection{Dystrophic MDX animal model}

The MDX mouse represents an established animal model of $\mathrm{X}$-linked muscular dystrophy [18]. In contrast to the relatively mild phenotype of MDX limb muscle, the MDX diaphragm exhibits severe fibre degeneration [17] and was therefore chosen in this study. A primary deficiency in the Dp427 isoform of the membrane cytoskeletal protein dystrophin, due to a point mutation in exon 23 [33], characterises this animal model of Duchenne/Becker's muscular dystrophy. Male mice of the Dmd ${ }^{\mathrm{MDX}}$ strain (Jackson Laboratory, Bar Harbor, Maine, USA) of varying age and age-matched C57 controls were obtained through the Animal House of the University of Bielefeld and the Bioresource Unit of the National University of Ireland, Maynooth. The Irish Higher Education Authority and the Deutscher Akademischer Austauschdienst generously supported laboratory visits to Germany by the Maynooth team. For the initial optimisation of the electrophoretic separation of crude diaphragm muscle samples and the subsequent comparative DIGE procedure, an animal population of 25 normal and 25 dystrophic mice was used. As previously shown by 1-D immunoblot analysis, the MDX mouse cohort used in this study lacks the $427 \mathrm{kDa}$ isoform of dystrophin and shows a drastic reduction in dystrophin-associated glycoproteins, such as dystroglycans and sarcoglycans [32]. According to international standards of DIGE analysis [9], six control samples, six MDX samples and six mixed standards were prepared for the statistical analysis of the electrophoretically separated muscle proteome using six DIGE gels. In addition, samples for the preparation of two pick gels were prepared, which were used in the mass spectrometric identification of individual 2-D diaphragm protein spots.

\subsection{Preparation and fluorescence labelling of muscle protein extracts}

Muscle specimens were quick-frozen in liquid nitrogen and ground up into a fine powder using a pestle and mortar. For comparative studies, equal amounts of normal and dystrophic diaphragm tissue ( 100 mg wet weight) were used. Muscle powder was resuspended in $1 \mathrm{~mL}$ of lysis buffer containing 9.5 M urea, 4\% w/v CHAPS, 0.5\% ampholytes $\mathrm{pH} 3-10$ and $100 \mathrm{mM}$ DTT. In order to eliminate excessive viscocity due to the presence of DNA, $2 \mu \mathrm{L}$ of DNase-I (200 units) were added per $100 \mu \mathrm{L}$ of extraction buffer [31]. Extraction buffer was also supplemented with a protease inhibitor cocktail $(0.2 \mathrm{mM}$ pefabloc, $1.4 \mu \mathrm{M}$ pepstatin, $0.15 \mu \mathrm{M}$ aprotinin, $0.3 \mu \mathrm{M}$ E-64, $1 \mu \mathrm{M}$ leupetin, $0.5 \mathrm{mM}$ soybean trypsin inhibitor, $1 \mathrm{mM}$ EDTA) to avoid proteolytic degradation of muscle proteins [20]. The suspension was incubated for $3 \mathrm{~h}$ at room temperature, with gentle vortexing every $10 \mathrm{~min}$ for $30 \mathrm{~s}$, and then centrifuged at $4^{\circ} \mathrm{C}$ for $20 \mathrm{~min}$ at $20000 \times \mathrm{g}$. The protein-containing middle layer was carefully removed and a portion of it was saved for the determination of protein concentration using the 2-D Quant kit from Amersham Biosciences/GE Healthcare. Since excess lipids, salts and nucleic acids may interfere with the proper 2-D separation of extracted proteins, and because other interfering contaminants may compete for the cyanine dyes, these substances were removed prior to IEF, using the 2-D Clean-Up kit from Amersham Biosciences/GE Healthcare. The precipitated protein fraction was resuspended in DIGE lysis buffer $(9.5 \mathrm{M}$ urea, 4\% w/v CHAPS, $30 \mathrm{mM}$ Tris-Cl, pH 8.5), mixed by gentle vortexing, pipetting and ultrasonnication, and then adjusted to a protein concentration of $1 \mathrm{mg} / \mathrm{mL}$ [32]. The $\mathrm{pH}$ value of the suspension was verified and, if needed, was adjusted using $30 \mathrm{mM}$ Tris$\mathrm{HCl}$. A $50 \mu \mathrm{g}$ protein sample was labelled for each of the three protein samples being studied, normal extracts were labelled with Cy3 dye, MDX samples with Cy5 dye and the pooled internal standard with Cy2 dye [9]. The labelling reaction was carried out for $30 \mathrm{~min}$ on ice and in the dark, and then quenched by incubation with $10 \mathrm{mM}$ lysine for $10 \mathrm{~min}$ on ice. The labelled protein extracts were pooled and immediately used for electrophoresis. An equal volume of $2 \times$ sample buffer (9.5 M urea, 4\% CHAPS, 2\% IPG Buffer pH 3-10 and $130 \mathrm{mM}$ DTT) was added and this suspension was left on ice for $10 \mathrm{~min}$ prior to electrophoretic separation.

\section{$2.42-D E$}

The 2-DE separation of fluorescently labelled muscle proteins was carried out by standard procedure using the recommended total protein concentration of $150 \mu \mathrm{g}$ protein per DIGE gel [7-9]. Using a reswelling tray from Amersham Biosciences/GE Healthcare, IPG strips pH 3-10 (linear) were rehydrated for $12 \mathrm{~h}$ with $0.45 \mathrm{~mL}$ of rehydration buffer (9.5 M urea, 4\% w/v CHAPS, 100 mM DTT, 1\% ampholytes pH 3-10), which had been complemented with $0.05 \%$ Bromophenol blue as a tracking dye. Following placement of the first-dimension strips gel-side up into the Ettan IPGphor manifold and coverage with $108 \mathrm{~mL}$ of drystrip cover fluid, protein samples were loaded by aniodic cup loading and were then run on the IPGphor IEF system from Amersham Biosciences/GE Healthcare. The following IEF gel running conditions were used: $180 \mathrm{~min}$ at $300 \mathrm{~V}$ (step-and-hold), $180 \mathrm{~min}$ at $600 \mathrm{~V}$ (gradient), $180 \mathrm{~min}$ at $1000 \mathrm{~V}$ (gradient), $180 \mathrm{~min}$ at $8000 \mathrm{~V}$ (gradient), $240 \mathrm{~min}$ at $8000 \mathrm{~V}$ (step-and-hold), $180 \mathrm{~min}$ at $500 \mathrm{~V}$ (step-and-hold) and $120 \mathrm{~min}$ at $8000 \mathrm{~V}$ (step-andhold). After IEF, the strips were equilibrated for $15 \mathrm{~min}$ in equilibration buffer (6 M urea, 30\% w/v glycerol, 2\% w/v SDS, 100 mM Tris-HCl, pH 8.8) containing 100 mM DTT and another $15 \mathrm{~min}$ in equilibration buffer supplemented with 0.25 M ideoacetamide. Using the Ettan Dalt-Twelve system from Amersham Biosciences/GE Healthcare, the 2-D separation of muscle proteins was performed with $12.5 \%$ resolving gels [32]. Following brief washing in SDS running buffer, IEF strips were positioned on top of the second-dimension slab gel with the help of a $1 \% \mathrm{w} / \mathrm{v}$ agrose sealing gel, poured on top of the strip and left to solidify. The six DIGE gels and the two pick gels were run for $0.2 \mathrm{~W} /$ gel for $1 \mathrm{~h}$, followed by $0.4 \mathrm{~W} /$ gel for $1 \mathrm{~h}$, and then ran at $1.5 \mathrm{~W}$ per gel overnight until the blue dye front had just disappeared from the bottom of the gel. 


\subsection{Gel image acquisition and image analysis}

Fluorescently labelled proteins were visualised using the Typhoon Trio variable mode imager system from Amersham Biosciences/GE Healthcare. Cy2, Cy3 and Cy5 images were scanned using a 488, 532 and $633 \mathrm{~nm}$ laser, respectively [9]. The PMT value for all scanned images was between 500 and $560 \mathrm{~V}$. All gels were scanned at $100 \mu \mathrm{m}$ resolution and, prior to analysis, gel images were cropped using the ImageQuant TL software programme [9]. Gel analysis was performed with the DeCyder V6.0 2-D analysis software package for DIGE (Amersham Biosciences/GE Healthcare). The DeCyder differential in-gel analysis (DIA) module was used for pairwise comparision of each normal and dystrophic MDX sample to the pooled standard present in each gel and for the calculation of normalised spot volume/protein abundance [9]. The 18-spor maps corresponding to the six gels were used to calculate average abundance and paired Student's $t$-test $p$ values for each protein across the six gels. This was done using the DeCyder biological variation analysis module (BVA) and Cy3:Cy2 and Cy5:Cy2 ratios for each individual protein. Protein spots that showed a 1.9-fold or more increase or decrease were then identified using mass spectrometric fingerprinting analysis.

\subsection{Mass spectrometric fingerprinting analysis}

Colloidal Coomassie staining, washing of gels, in-gel digestion and MALDI-TOF MS analysis were carried out as previously described in detail [20,31]. Excised gel plugs of interest were placed in $1.5 \mathrm{~mL}$ Eppendorf tubes that had been presilconised with Sigmacote (Sigma Chemical Company) and then destained, desalted and washed by standard procedure [31]. Gel pieces were rehydrated with digestion buffer, containing $1 \mu \mathrm{g}$ of trypsin (Promega) per $20 \mu \mathrm{L}$ of $50 \mathrm{mM} \mathrm{NH} \mathrm{HCO}_{3}$, so that individual gel plugs were sufficiently covered [32]. Following an incubation step for $60 \mathrm{~min}$ at $37^{\circ} \mathrm{C}$, excess enzyme solution was removed and $3 \mu \mathrm{L}$ of $50 \mathrm{mM} \mathrm{NH} \mathrm{HCO}_{3}$ was added to each gel plug in order to keep gel pieces wet overnight. Samples were then centrifuged at $12000 \times \mathrm{g}$ for $10 \mathrm{~min}$ and the resulting supernatant was carefully removed from each incubation tube and placed into a clean and silconised plastic tube. To aid digestion, the gel plugs were treated by three sequential extraction steps, as previously outlined [32], and then the extracted solutions combined, concentrated and resuspended in $8 \mu \mathrm{L}$ of $3 \% \mathrm{v} / \mathrm{v}$ TFA and stored at $-70^{\circ} \mathrm{C}$ until analysed by MS. MALDI-TOF MS tryptic PMF of in-gel digests from normal versus dystrophic MDX muscle protein species was carried out by an optimised procedure [31, 32]. Following desalting with Millipore C-18 ZipTips, tryptic peptides from individual 2-D muscle protein spots were eluted onto the sample plate with the matrix solution, consiting of $5 \mathrm{mg} / \mathrm{mL}$ CHCA in $50 \% \mathrm{v} / \mathrm{v}$ ACN $/ 0.1 \% \mathrm{v} / \mathrm{v}$ TFA. An Ettan MALDI-TOF Pro instrument from Amersham Biosciences was used for the recording of mass spectra, operating in the positive reflector mode at the following parameters: accelerating voltage $20 \mathrm{kV}$; and pulsed extraction: on (focus mass 2500). For internal calibration, standard peaks of angiotensin III $(897.5 \mathrm{~m} / \mathrm{z})$ and ACTH $(2465.19 \mathrm{~m} / \mathrm{z})$, as well as trypsin autolysis peaks at 842.50 and $2211.104 \mathrm{~m} / z$, were used. For the analysis of mass spectra, MALDI evaluation software was employed and for the identification of protein species, the PMF ProFound search engine for peptide mass fingerprints was used. All certainty hits of diaphragm muscle proteins generated by the ProFound search engine were matched against the publicly available search engine MASCOT (http://www.matrixscience.com).

\subsection{Immunoblot analysis}

2-DE separated muscle proteins were transferred at $100 \mathrm{~V}$ for $80 \mathrm{~min}$ to Immobilin NC-pure NC membranes using a Transblot Cell from BioRad Laboratories (Hemel Hempstead, Herts., UK) according to Towbin et al. [34] Ponceau-SRed staining of NC membranes was employed to evaluate the efficiency of the electrophoretic transfer, followed by destaining in PBS $(50 \mathrm{mM}$ sodium phosphate, $0.9 \% \mathrm{w} / \mathrm{v}$ $\mathrm{NaCl}, \mathrm{pH}$ 7.4). NC sheets were blocked for $1 \mathrm{~h}$ in $5 \% \mathrm{w} / \mathrm{v}$ fatfree milk powder, dissolved in PBS. Incubation with sufficiently diluted primary antibodies and peroxidase-conjugated secondary antibodies, as well as washing steps, were performed as previously described in detail [35]. Immunodecorated 2-D protein spots were visualised using the SuperSignal ECL kit from Pierce and Warriner. Densitometric scanning of immunoblots was performed on a Molecular Dynamics 300S computing densitometer (Sunnyvale, CA, USA) with Imagequant V3.0 software.

\subsection{Immunofluorescence microscopy}

Cryosections of $10 \mu \mathrm{m}$ thickness were prepared on a Thermo Shandon Cryotome (Life Science International, Cheshire, UK) and mounted on Superfrost Plus positively charged microscope slides. For better handling and optimum sectioning, individual diaphragms were carefully rolled into a tube-like specimen, and then quick-frozen, fixed and blocked as previously described [35]. Sections were cut at a slight angle to expose both a transfer and longitudinal direction. Cryoections were incubated overnight at $4^{\circ} \mathrm{C}$ with primary antibodies, carefully washed [35], and then incubated for $30 \mathrm{~min}$ at $25^{\circ} \mathrm{C}$ with secondary antibodies that had been conjugated to Alexa Fluor 488 dye or Alexa Fluor 594 dye. Image acquisition was performed with an Olympus FluoView FV1000 confocal laser scanning microscope (Olympus Life and Material Science Europe, Hamburg, Germany) using the Olympus FluoView Version 1.3c software package. In order to establish the dystrophic status of MDX specimens, normal and MDX cryosections were labelled with antibodies to the dystrophin-associated glycoprotein $\beta$-dystroglycan, using Alexa Fluor 594 dye. The subcellular localisation of the small heat shock protein cvHSP was carried out 
with Alexa Fluor dye 488. For labelling the position of nuclei within muscle fibres, cryosections were incubated with the DNA-binding dye diamidino-phenyindole [35].

\section{Results}

\subsection{Sensitivity of DIGE technique for the detection of diaphragm proteins}

2-DE represents an extremely powerful biochemical technique for the separation of proteins. Using standard 2-D gel approaches with conventional staining techniques, the total number of detectable spots is usually in the range of several 100 proteins. In contrast, advanced DIGE, using the CyDye DIGE fluor dyes Cy2, Cy3 and Cy5 for the labelling of pooled standards, control samples and diseased samples, respectively, can decisively increase the detection levels for comparative proteomics studies [9]. Although skeletal muscle fibres are a relatively difficult tissue to be properly separated by gel electrophoresis, in this study the application of the DIGE technique resulted in the detection of 2398 individual 2-D muscle protein spots. Previous studies on the 2-D separation of skeletal muscle proteins have reported 333 Hot Coomassiestained protein spots [32] and 652 Silver-stained protein spots [5]. Consequently the DIGE analysis presented here shows an approximately 3.7- to 7.2-fold increase in its protein detection rate as compared to conventional staining techniques. Thus, although certain diaphragm muscle species may still be under-represented in the master gel shown in Fig. 1, and the majority of the diaphragm proteins seen are probably mostly soluble elements of muscle fibres or at least proteins that do not exist in detergent-insoluble membrane complexes, the DIGE approach exhibited a greatly enhanced detection rate.

\subsection{Severely disturbed protein expression pattern in dystrophin-deficient diaphragm}

The comparative gel electrophoretic analysis of the normal versus the MDX diaphragm proteome, using a Typhoon Trio variable imager and DeCyder 2-D analysis software, revealed that out of 2398 detectable 2-D protein spots, 35 proteins showed a drastic differential expression pattern. Since no specific diaphragm 2-D standard gel exists in the Swiss-2-D PAGE mouse tissue databank, the spot pattern of our diaphragm gel (Fig. 1) was compared to the international mouse limb muscle database (http://www.us.expasy.org/ch2d/). The overall 2-D spot pattern of the master gel was found to be comparable to published studies on the electrophoretic separation of total skeletal muscle extracts [5, 6]. As illustrated in Fig. 1, protein species with a changed abundance in MDX diaphragm ranged in molecular mass from $6.2 \mathrm{kDa}$ ( $\alpha$-globin) to $223.7 \mathrm{kDa}$ (myosin heavy chain) and covered a $\mathrm{pI}$ range from pI 4.9 (vimentin) to $\mathrm{p} I 9.2$ (isocitrate dehydrogenase). An increased expression was found in the case of 14 muscle proteins, and 21 diaphragm proteins were shown to be

\section{DIGE Cy2 Master Gel of Diaphragm Muscle}

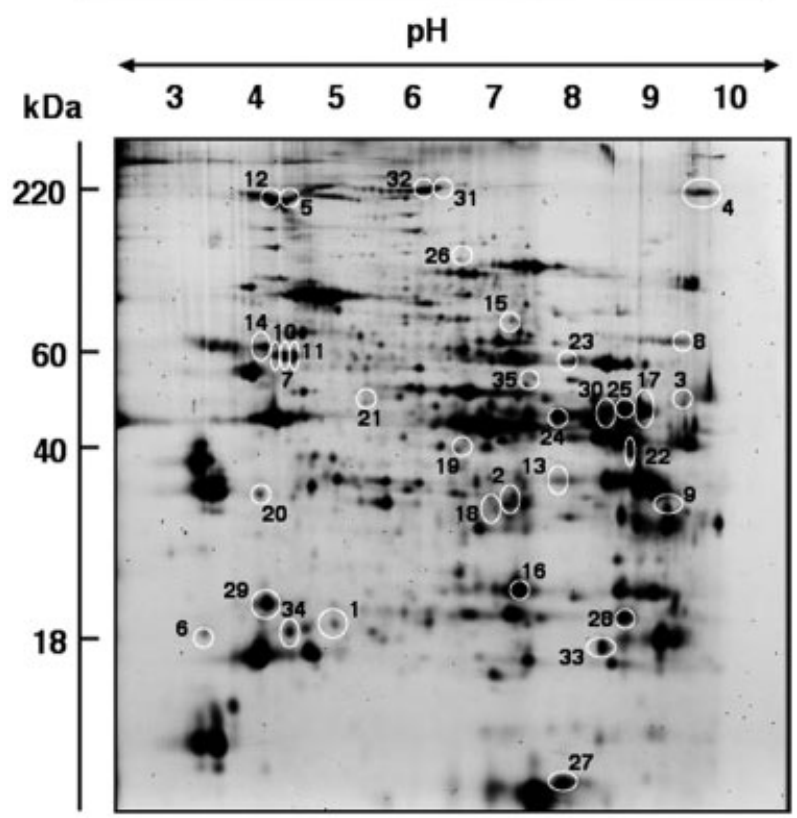

Figure 1. 2-DE analysis of normal vs. MDX diaphragm muscle extracts using DIGE analysis. Shown is a Cy2-labelled master gel of total protein extracts from normal and dystrophic MDX diaphragm muscle. The $\mathrm{pH}$ values of the first dimension gel system and molecular mass standards (in $\mathrm{kDa}$ ) of the second dimension are indicated on the top and on the left of the panels, respectively. Proteins with a drastically different expression level are marked by circles and are numbered 1-35. See Table 1 for a detailed listing of diaphragm muscle proteins with a changed abundance in dystrophin-deficient fibres.

decreased in their abundance. Table 1 lists the identification of the majority of these proteins using MALDI-TOF MS tryptic PMF from in-gel digests. Proteins with a disturbed expression pattern belonged to the class of cellular components that are involved in muscle contraction, cytoskeleton formation, mitochondrial function, metabolism, ion homeostasis and chaperone function. Diaphragm proteins of increased abundance in the MDX animal model could be grouped as components involved in chaperone function (cardiovascular heat shock protein/spot 1; chaperonin/ spot 11), aldehyde metabolism (aldehyde reductase/spot 2), intermediate filament formation (desmin/spot 7; vimentin/ spot 10), muscle contraction (myosin heavy chains/spot 5 and 12), glycolysis (glyceraldehyde-3-phosphate dehydrogenase/spot 9), fatty acid oxidation (electron transferring flavoprotein/spot 13) and nucleotide metabolism (Atp5b protein/spot 14). The presence of the slow-twitch type I/cardiac myosin heavy chain isoform (spot 5) clearly revealed the special status of the diaphragm muscle within the various skeletal muscle fibre groupings of contractile mouse tissues. Diaphragm proteins of decreased abundance in the MDX animal model could be grouped as components involved in 
Table 1. List of DIGE-identified muscle proteins that exhibit a drastic change of expression in dystrophin-deficient MDX diaphragm

\begin{tabular}{|c|c|c|c|c|c|c|}
\hline $\begin{array}{l}\text { Spot } \\
\text { no. }\end{array}$ & Name of identified proteins & $\begin{array}{l}\text { Sequence } \\
\text { coverage } \\
(\%)\end{array}$ & $\begin{array}{l}\text { Molecular } \\
\text { mass } \\
(\mathrm{kDa})\end{array}$ & $\mathrm{p} /$ & $\begin{array}{l}\text { Protein } \\
\text { accession } \\
\text { no. }\end{array}$ & $\begin{array}{l}\text { Fold- } \\
\text { change } \\
+/-\end{array}$ \\
\hline 1 & Heat shock protein cvHSP & 21.3 & 18.66 & 5.8 & gi[6636001] & +8.09 \\
\hline 2 & Aldehyde reductase & 18.2 & 36.80 & 6.9 & gi[29374169] & +3.82 \\
\hline 3 & Unknown protein & - & - & - & - & +3.05 \\
\hline 4 & Unknown protein & - & - & - & - & +2.82 \\
\hline 5 & Cardiac myosin heavy $\beta$-chain & 10.5 & 223.66 & 5.6 & gi[16508127] & +2.43 \\
\hline 6 & Unknown protein & - & - & - & - & +2.23 \\
\hline 7 & Desmin & 24.9 & 53.54 & 5.2 & gi[33563250] & +2.14 \\
\hline 8 & Unknown protein & - & - & - & - & +2.14 \\
\hline 9 & Glyceraldehyde-3-phosphate dehydrogenase-like protein & 33.6 & 36.09 & 8.4 & gi[51710824] & +2.12 \\
\hline 10 & Vimentin & 20.2 & 51.60 & 4.9 & gi[2078001] & +2.10 \\
\hline 11 & Chaperonin, HSP-1 & 23.4 & 61.11 & 5.7 & gi[31981679] & +2.00 \\
\hline 12 & Muscle myosin heavy chain & 4.8 & 223.77 & 5.6 & gi[71143152] & +1.97 \\
\hline 13 & Electron transferring flavoprotein, $\alpha$-polypeptide & 34.2 & 35.27 & 8.8 & gi[31981826] & +1.96 \\
\hline 14 & Atp5b protein & 27.0 & 56.28 & 5.2 & gi[23272966] & +1.93 \\
\hline 15 & Jmjd1a protein & 10.3 & 67.61 & 6.6 & gi[20071828] & -1.91 \\
\hline 16 & Carbonic anhydrase 3 & 28.1 & 29.63 & 6.9 & gi[13786200] & -1.92 \\
\hline 17 & Isocitrate dehydrogenase 2 & 10.0 & 51.34 & 9.2 & gi[37748684] & -1.95 \\
\hline 18 & Unknown protein & - & - & - & - & -1.95 \\
\hline 19 & Sorbitol dehydrogenase & 28.0 & 40.64 & 6.6 & gi[25108890] & -1.97 \\
\hline 20 & Regucalcin & 37.8 & 33.90 & 5.2 & gi[6677739] & -1.98 \\
\hline 21 & Alpha actin (aa27-375) & 15.8 & 39.46 & 5.8 & gi[49870] & -2.13 \\
\hline 22 & Betaine-homocysteine methyltransferase & 19.7 & 45.46 & 8.4 & gi[62533211] & -2.14 \\
\hline 23 & Aldh2 protein & 18.1 & 57.03 & 7.7 & gi[13529509] & -2.15 \\
\hline 24 & Creatine kinase, mitochondrial & 31.3 & 47.91 & 9.1 & gi[38259206] & -2.17 \\
\hline 25 & Aldolase 1, A-lsoform & 25.5 & 39.75 & 8.9 & gi[42490830] & -2.31 \\
\hline 26 & Unknown protein & - & - & - & - & -2.56 \\
\hline 27 & Alpha-globin & 69.0 & 6.20 & 6.8 & gi[193761] & -2.65 \\
\hline 28 & Troponin I, fast muscle, Tnl-2 & 37.4 & 21.51 & 8.8 & gi[6678391] & -2.65 \\
\hline 29 & Myosin light chain & 29.4 & 22.52 & 5.0 & gi[33563264] & -2.77 \\
\hline 30 & Dihydrolipoamide dehydrogenase & 20.4 & 54.76 & 8.3 & $\operatorname{gi}[6014973]$ & -2.79 \\
\hline 31 & Unknown protein & - & - & - & - & -2.80 \\
\hline 32 & Unknown protein & - & - & - & - & -3.08 \\
\hline 33 & Beta haemoglobin & 54.1 & 15.76 & 7.3 & gi[229301] & -3.24 \\
\hline 34 & Adenylate kinase 1 & 31.9 & 23.33 & 5.7 & gi[10946936] & -3.24 \\
\hline 35 & F-box protein Fbxo11 & 10.4 & 58.58 & 6.6 & gi[33243977] & -3.52 \\
\hline
\end{tabular}

protein ubiquination (Fbxo11 protein/spot 35), nucleotide metabolism (adenylate kinase/spot 34; creatine kinase/ spot 24), oxygen transport ( $\beta$-haemoglobin/spot 33; $\alpha$-globin/spot 27), mitochondrial enzymes associated with the formation of acetyl-coenzyme A (dihydrolipoamide dehydrogenase/spot 30), muscle contraction (myosin light chain/ spot 29; troponin/spot 28; actin/spot 21), glycolysis (aldolase/spot 25), aldehyde metabolism (aldehyde dehydrogenase/spot 23), the remethylation pathway of homocysteine homeostasis (betaine-homocysteine methyltransferase/spot 22), cytosolic calcium homeostasis (regucalcin/ spot 20), the polyol pathway of glucose metabolism (sorbitol dehydrogenase/spot 19), the citric acid cycle (isocitrate dehydrogenase/spot 17), the regulation of acid-base balance (carbonic anhydrase/spot 16) and transcriptional control (jumonji-domain containing Jmjd1a protein/spot 15).

\subsection{Drastic increase of the heat shock protein cvHSP in the dystrophic MDX diaphragm}

The cardiovascular heat shock protein, cvHSP of $18.7 \mathrm{kDa}$ and $\mathrm{p} I 5.8$, exhibited the highest fold change in expression of the 35 detected diaphragm proteins. As shown in the enlargements of Cy3-, Cy2- and Cy5-labelled gels (Fig. 2A-C), two distinct protein spots positioned in close vicinity to the myosin light chain isoforms represent the enzyme adenylate kinase and cvHSP. In diaphragm, the apparent molecular mass and $\mathrm{p} I$ value of myosin light chain isoforms MLC-3 (gi33563264) and MLC-2v (gi38511915) are $22.5 \mathrm{kDa} / \mathrm{p} I 5.0$ and $18.8 \mathrm{kDa} / \mathrm{pI} 4.9$, respectively. This correlates relatively well with their position in the 2-D master gel (Fig. 1) and appears to be different to gastrocnemius muscle where some myosin light chains exhibit a slightly abnormal electropho- 

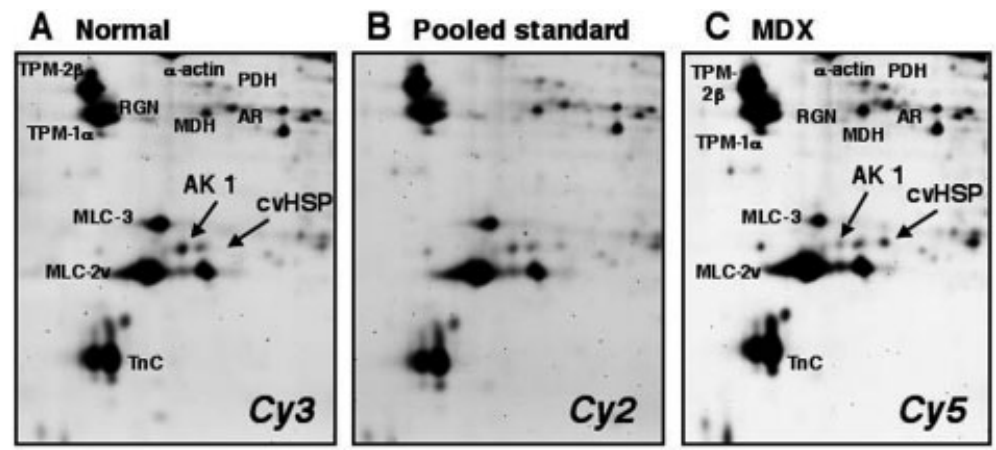

Figure 2. Differential expression pattern of the small heat shock protein cvHSP in normal vs. MDX diaphragm muscle. Shown is an expanded view of 2-D gels of normal diaphragm (A), pooled standard (B) and dystrophic MDX diaphragm (C) from which the increased expression levels of the cardiovascular heat shock protein (cvHSP; Protein Accession No. gi[6636001]) in MDX preparations can be observed. In contrast, the myosin light chain isoform MLC-3, the cytosolic $\mathrm{Ca}^{2+}$-binding protein regucalcin (RGN) and the enzyme adenylate kinase (AK1) are decreased in MDX diaphragm. Proteins in panels (A)-(C) were labelled with the CyDye DIGE fluor dyes Cy3, Cy2 and Cy5, respectively. Major 2-D landmark proteins of unchanged abundance are represented by the two tropomyosin isoforms TPM-1 $\alpha$ and TPM-2 $\beta$, troponin C (TnC), myosin light chain MLC-2v, $\alpha$-actin, pyruvate dehydrogenase (PDH), aldehyde reductase (AR) and malate dehydrogenase (MDH). The portion of the 2-D gel (see Fig. 1) shown covers the range of approximately pH 3-6 in the first dimension and a molecular mass range of approximately $10-40 \mathrm{kDa}$ in the second dimension.

retic mobility pattern [6]. While the adenylate kinase spot is drastically decreased in MDX gels, the cvHSP spot increased dramatically in dystrophin-deficient preparations. It has recently been reported that adenylate kinase and the $\mathrm{Ca}^{2+}$. binding protein regucalcin are both reduced in MDX skeletal muscle using conventional proteomics [30, 32]. This agrees with the findings represented in Fig. 2, whereby the regucalcin spot is positioned close to the tropomyosin TPM- $1 \alpha$ spot. Abundant landmark 2-D protein spots representing two tropomyosin isoforms, actin, pyruvate dehydrogenase, aldehyde reductase and malate dehydrogenase showed a relatively comparable expression level in normal versus MDX diaphragm preparations. This confirms the reproducibility of comparative approaches that employ DIGE analysis and shows the significance of the drastic increase in the small heat shock protein of $18.7 \mathrm{kDa}$.

\subsection{Immunoblotting confirms increased cvHSP expression in MDX diaphragm}

Application of the DeCyder software programme for the DIGE analysis of normal versus dystrophic MDX diaphragm preparations revealed an approximate eight-fold increase in protein spot 1 (Table 1). This finding is clearly illustrated in the enlarged gel panel and its comparative graphic representation of the cvHSP spot in Fig. 3A. To confirm this finding, immunoblotting with a polyclonal antibody against a synthetic peptide that corresponds to amino acids 150 to 166 of the human crHSP protein, was performed. Since this stretch of peptide sequence has $100 \%$ homology to mouse cvHSP, antibody ab21128 to this small heat shock protein represented the ideal tool to highly specifically detect the immobilised mouse antigen. 2-D immunoblot analysis con- firmed the drastic increase of cvHSP in the dystrophic MDX diaphragm (Fig. 3B). The expression of the small heat shock protein was shown to be approximately three-fold increased in 9-wk-old MDX diaphragm and more than seven-fold increased in 11-month-old dystrophic fibre preparations.

\subsection{Immunofluorescence microscopy shows increased cvHSP expression in MDX diaphragm}

The biochemical findings from the immunoblotting of cvHSP were confirmed by confocal laser scanning microscopy, as illustrated in Fig. 4, using antigen labelling with Alexa Fluor 488 dye-conjugated secondary antibodies to primary anti-cvHSP antibodies. As compared to normal skeletal muscle, dystrophin-deficient MDX diaphragm fibres clearly exhibited a drastically reduced expression level of the dystrophin-associated glycoprotein $\beta$-dystroglycan (Fig. 4A, D), which has been shown previously $[14,16]$, and an increased abundance of the heat shock protein cvHSP (Fig. 4C, F). The position of nuclei within normal and dystrophic muscle fibres was marked by the DNA-binding dye diamidino-phenyindole (Fig. 4B, E). The combined images of Fig. 4G (normal) versus Fig. 4H (MDX), showing red fluorescence labelling for $\beta$-dystroglycan, green fluorescence labelling for cvHSP and blue fluorescence labelling for nuclei, outline the sarcolemmal localisation of the dystrophin-associated glycoprotein and the mostly cytoplasmic localisation of the small heat shock protein. The concentration of cvHSP was found to be relatively low in normal fibres and appeared to be concentrated in subsarcolemmal regions. In stark contrast, all fibres investigated in the MDX diaphragm exhibited a high density of this heat shock protein throughout the cytoplasmic region, with a typical striated appearance in the 


\section{A DIGE analysis of cuHSP}
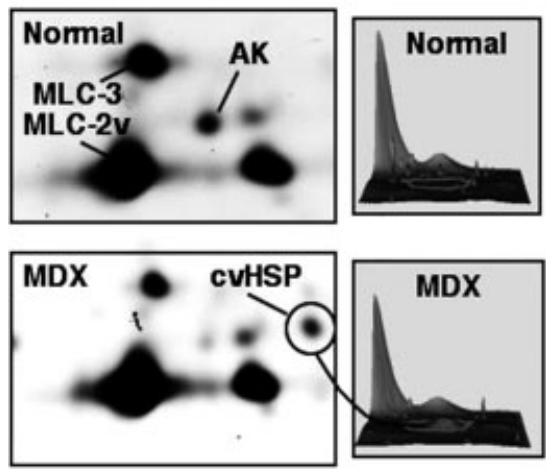

B 2-D Immunoblot analysis of cuHSP

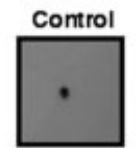

9W-MDX

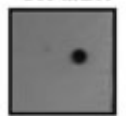

$11 \mathrm{~m} \rightarrow \mathrm{MDX}$
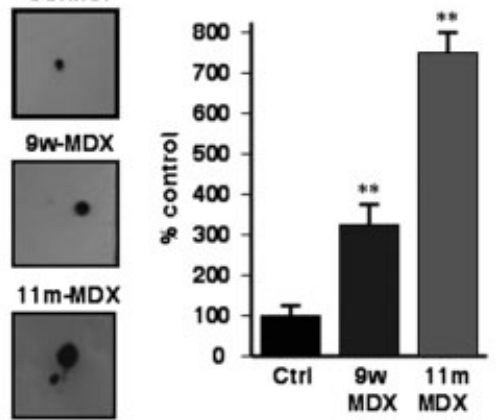

Figure 3. Immunoblot analysis of the small heat shock protein cvHSP in normal vs. MDX diaphragm muscle. Shown is a comparison of the results obtained with DIGE analysis (A) vs. immunoblotting $(B)$ to determine the differential expression levels of the small heat shock protein cvHSP in normal vs. dystrophic MDX diaphragm. Panel (A) illustrates the eight-fold increase of the cvHSP spot of $18.7 \mathrm{kDa}$ and $\mathrm{p} / 5.8$ in MDX preparations (see Table 1). Shown is an expanded view of the Cy3- and Cy5-labelled 2-D gels of normal and dystrophic diaphragm, respectively, and the comparative graphic representation of the cvHSP spot. Immunoblotting with polyclonal antibody ab21128 against a synthetic peptide that corresponds to amino acids $150-166$ of the human/mouse cvHSP protein documents an approximately three-fold increase in 9-wk-old MDX diaphragm and a more than seven-fold increase in 11-month-old dystrophic fibre preparations ( $n=6$; ${ }^{*} p<0.01$; unpaired Student's $t$-test).

longitudinal direction. This indicates a potential association of cvHSP with contractile elements and/or cytoskeletal components, and could therefore play a role in the stress response to a damaged membrane cytoskeletal network in dystrophin-deficient fibres.

\subsection{Immunoblotting survey of key heat shock proteins in MDX diaphragm}

Based on the DIGE-generated finding that crHSP is increased in MDX diaphragm, it was of interest to survey the fate of other key heat shock proteins in dystrophin-deficient fibres. Figure 5A shows representative 2-D immunoblots of

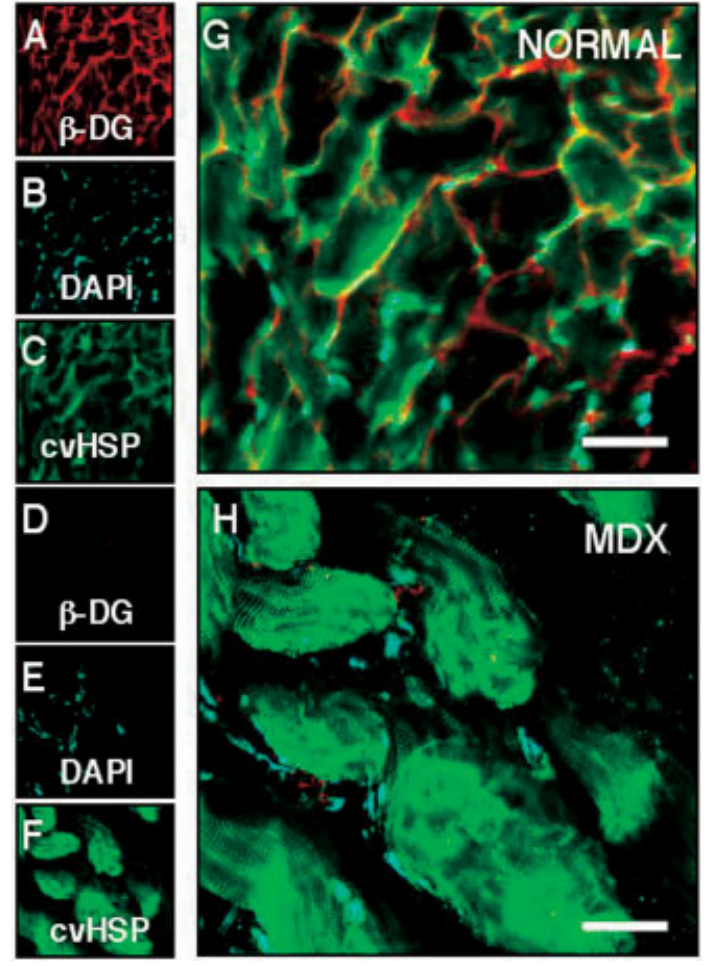

Figure 4. Immunofluorescence localisation of cvHSP in normal vs. MDX diaphragm. Shown is the confocal laser scanning microscopical analysis of 11-month-old normal (A-C, G) vs. agematched MDX (D-F, H) diaphragm cryosections. The dystrophinassociated glycoprotein $\beta$-dystroglycan ( $\beta$-DG; $A, D, G, H$ ) and the cardiovascular heat shock protein (cvHSP; C, F, G, H) were labelled with antibody-conjugated Alexa Fluor 594 dye and Alexa Fluor 488 dye, respectively. The position of nuclei within normal and MDX muscle fibres was marked by the DNA-binding dye diamidino-phenyindole (DAPI; B, E, G, H). The immunofluorescence images indicate that sarcolemmal $\beta$-dystroglycan levels are decreased in MDX diaphragm and that the cytosolic abundance of cvHSP is drastically increased in dystrophin-deficient fibres. Panels $A-F$ show individual fluorescence images, while panels $\mathrm{G}$ and $\mathrm{H}$ show combined fluorescence images. Bar equals $40 \mu \mathrm{m}$.

the main subclasses of muscle heat shock proteins, and Fig. 5B illustrates graphically the expression pattern of these chaperones in normal versus dystrophic fibres. HSP25, HSP60 and HSP70 appear not to be drastically changed in their abundance in MDX diaphragm. No statistical analysis is shown for HSP70 in Fig. 5B due to difficulties with high background staining following antibody decoration, which prevented reliable densitometric scanning. HSP20, GRP75 and HSP90 were shown to be reduced in dystrophin-deficient fibres. In analogy to cvHSP, the large heat shock protein HSP110 was drastically increased in its expression in MDX diaphragm preparations. Our immunoblotting survey of the various representative heat shock proteins indicates a differential expression pattern of key chaperones during muscle degeneration in X-linked muscular dystrophy. 


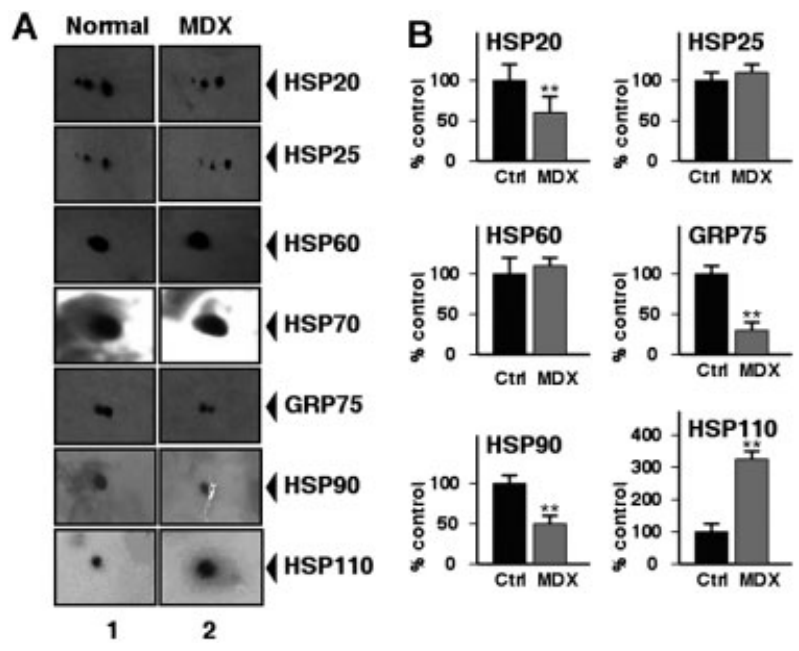

Figure 5. Immunoblotting survey of key heat shock proteins in the MDX diaphragm. Shown is the 2-D immunoblot analysis of heat shock proteins HSP20, HSP25, HSP60, HSP70, GRP75, HSP90 and HSP110, whereby lanes 1 and 2 represent 9-wk-old normal and age-matched dystrophic preparations, respectively (A). Panel (B) shows the comparative graphic representation of immunodecoration with antibodies to the various subclasses of muscle heat shock proteins $\left(n=6\right.$; ${ }^{*} p<0.01$; unpaired Student's $t$-test). Due to problems with high levels of background staining, no statistical evaluation of HSP70 is shown.

\section{Discussion}

Although prefractionation methods exist that can be employed to remove abundant structural proteins prior to the 2-D separation of muscle proteins [36], comparative proteomics approaches usually try to avoid fractionation schemes in order to achieve a true representation of the skeletal muscle proteome from normal versus diseased specimens [3]. By their very nature, extraction procedures and centrifugation steps may introduce artefacts. We therefore used total tissue preparations in order to investigate the entire accessible proteome of control versus MDX diaphragm. The list of 35 DIGE-identified diaphragm proteins that exhibited a drastic change of abundance in MDX fibres indicates that a deficiency in the membrane cytoskeletal protein dystrophin triggers a severely disturbed protein expression pattern in muscular dystrophy. Although the initial pathobiochemical consequence of a primary genetic abnormality in the Dp427 isoform is clearly a drastic reduction in dystrophin-associated glycoproteins and associated surface components $[11,14,16]$, this impairment of sarcolemmal integrity appears to trigger a variety of downstream changes in various cellular elements. The pathophysiological up- or downregulation in dystrophic fibres includes key diaphragm proteins that are involved in chaperone function, ion homeostasis, muscle contraction, cytoskeleton formation and metabolism.

Various metabolic marker components, such as proteins associated with aldehyde metabolism, glycolysis, the citric acid cycle, fatty acid oxidation, oxygen transportation, nucleotide metabolism, the formation of acetyl-coenzyme and the remethylation pathway are differentially affected in the MDX diaphragm. In addition, important physiological regulators that influence acid-base balance and cytosolic calcium binding, are changed in dystrophin-deficient fibres. The loss of a proper linkage between the actin membrane cytoskeleton and the extracellular matrix in the dystrophic plasmalemma seems to result in major disturbances in fibre homeostasis and muscle metabolism, which encompasses both the compensatory up-regulation of key enzymes and the loss of other metabolic regulators. Microrupturing of the dystrophic surface membrane has previously been implicated in causing disturbed ion fluxes [37, 38]. Besides abnormal ion cycling in dystrophic fibres, a reduction in overall cellular integrity may also cause disturbed fluxes of metabolic substrates and protein factors [39] and thereby negatively influence the regulation of important pathways. Interestingly, the decreased abundance in the Fbxo11 protein involved in ubiquination agrees with studies that have shown an impaired ubiquitin-proteasome system in muscle wasting processes [40]. The approximate three-fold reduction of the enzyme adenylate kinase in MDX diaphragm, as shown here by DIGE analysis, has recently been shown in dystrophic MDX hind limb fibres by conventional proteomics [30]. In conjunction with creatine kinase, adenylate kinase provides a major nucleotide pathway, and the lack of this key enzyme indicates that an abnormal regulation of nucleotide ratios occurs in dystrophic muscles. This appears to be a major metabolic defect in dystrophinopathy.

Many potential biological and technical factors can complicate the comparative proteomic analysis of total muscle extracts, such as marked changes in the ratio of connective to muscle fibres, alterations in the interstitial volume, residual blood components or drastic shifts in fibre types. Hence, changes in the abundance of contractile proteins, such as actin and myosin isoforms, are difficult to judge. Degradation of the contractile apparatus should result in a comparable loss and/or compensatory increase in the main contractile components. However, such an analysis is complicated by the presence of proteolytic fragments and the potential aggregation of large muscle proteins during the electrophoretic separation procedure. The DIGE analysis presented here indicates a general disturbance of myosin isoforms and actin, but it is not possible to make definitive conclusions about fibre shifting in the dystrophin-deficient diaphragm. Our recent proteomic analysis of the fast-to-slow fibre transition process in chronic low-frequency stimulated tibialis anterior muscle revealed distinct switches in troponin and myosin isoforms [20]. Such drastic alterations in contractile elements have not been observed in the MDX muscle investigated here. Interestingly, it has previously been reported that adult MDX muscle exhibits a wild-type pattern of myosin heavy chain isoform expression [41]. This strongly argues against a significant pathological fibre type shift in the MDX animal model, and suggests that ongoing physical activity, rather than the developmental history of a muscle, is 
the key determinant of myosin isoform expression. No marked changes in the expression of albumin have been shown by the DIGE analysis of the MDX diaphragm indicating that the relative volume and composition of the interstitial space is probably comparable between normal and dystrophin-deficient fibres. In contrast, the increased extracellular space in conditioned muscle fibres was recognised by a four-fold increase in albumin levels, as recently judged by comparative proteomics [20]. This appears not to be the case in the MDX diaphragm.

The increased sensitivity of the DIGE technique detected, besides the previously identified changes in adenylate kinase [30] and regucalcin [32], a drastic increase in the large heat shock protein chaperonin and an important small heat shock protein, cvHSP. Since the up-regulation of stress factors is an essential response to cellular injury in many pathological processes, this new result is a significant finding for our understanding of muscular dystrophy. Besides heat shock, diverse conditions such as inflammation, ischemia, oxidative stress, exposure to heavy metals or certain amino acid analogues, trigger the rapid synthesis of the highly conserved group of heat shock proteins [42]. The multigene family of HSP proteins functions mostly as chaperons [43]. They are essential for normal cellular activity by associating with malfolded proteins, thereby preventing their aggregating into large deleterious complexes. HSP proteins enable cells to survive stressful conditions by aiding the renaturation process of malfolded proteins, but are also involved in influencing the intracellular transport of mature proteins. In addition to high-molecular mass heat shock proteins, a subfamily of ten small heat shock proteins (smHSPs) exists in mammalian species [44], including the cardiovascular heat shock protein cvHSP [45]. Members of the smHSP class of proteins range in molecular mass from 15 to $30 \mathrm{kDa}$. They are characterised by a homologous domain of approximately 100 amino acids, the so-called $\alpha$-crystallin domain [46]. Interestingly, the smHSP group of proteins mediates mostly the stabilisation of cytoskeletal protein folding and transportation [46]. Thus, the up-regulation of cvHSP in a cytoskeletal disease process, which is clearly present in dystrophinopathies, suggests that damaged muscle fibres attempt to rescue their cellular integrity by modulating and repairing the cytoskeletal network.

It has previously been reported in heart muscle that the smHSP called $\alpha$ B-crystallin interacts with actin, desmin and vimentin during biological stress [47]. A key function of smHSPs appears to be the stabilisation of cytoskeletal structures, which is essential for cellular survival. This agrees with our immunofluorescence analysis of cvHSP in MDX diaphragm fibres, which indicates that this heat shock protein may be associated with cytoskeletal elements or the contractile apparatus. In addition, the increased levels of desmin and vimentin in the MDX diaphragm, as also identified in this DIGE-based study, could present a compensatory mechanism of the dystrophic fibre. Vimentin and desmin may strengthen the overall cellular integrity of the muscle fibre by partially substituting for the loss of the membrane cytoskeletal element dystrophin. Possibly, the load-bearing function of the skeletal muscle cytoskeleton is rescued by the up-regulation of the proteins that form the structural backbone of intermediate filaments. Changes in cvHSP have probably not been detected previously because of the lack of sensitivity in detection levels of proteomics approaches that employ conventional staining techniques, such as Hot Coomassie or Silver staining. This clearly shows the advantage of using DIGE-based approaches in comparative proteomics [7-9].

Our finding that cvHSP, chaperonin and HSP-110 expression is increased in dystrophin-deficient fibres indicates that the dystrophic fibre degeneration process appears to trigger the up-regulation of specific chaperones that directly interact with and stabilise malfolded or damaged muscle proteins. A previous immunohistochemical study showed a changed expression pattern of large heat shock proteins in dystrophin-deficient fibres [48]. HSP-65, HSP-72 and HSP-72/73 were shown to be significantly induced in hypercontracted fibres [48]. Hypercontraction of fibres is a specific histopathological feature of dystrophin-deficient muscles [2] and is associated with a high frequency of cytosolic $\mathrm{Ca}^{2+}$-loading $[12,49]$. Increased cellular $\mathrm{Ca}^{2+}$-levels cause a pathological cycle of muscle protein degradation and contractile weakness rendering fibres more susceptible to necrosis [50]. In agreement with the cellular analysis of heat shock protein expression in dystrophic muscles by Bornman et al. [48], the proteomic profiling shown here suggests that the induced stress response relate, at least partially, to the metabolic abnormalities triggered by the deficiency in the membrane cytoskeletal protein dystrophin. In addition, the immunofluorescence localisation of cvHSP to the subsarcolemmal cytosol in normal fibres and its drastically increased density throughout the cytosplasm in MDX diaphragm, also indicates an important protective role of this small heat shock protein in muscle fibre degeneration.

Previous studies have established that seven of the ten identified small heat shock proteins are preferentially expressed in striated muscle tissues and that they provide a complex stress response system in skeletal muscle [44, 51]. An ischemia-induced stress response triggers the rapid translocation of all small heat shock proteins to myofibrillar targets in the Z/I-area of muscle fibres [44]. The 2-D immunoblot analysis presented here showed a differential response of small heat shock proteins in muscular dystrophy. HSP-20 expression was reduced, cvHSP expression increased, and HSP25 expression relatively unchanged. To fully understand these alterations in smHSP abundance, it is essential to know their exact molecular-binding sites, which has not yet been established [51]. Most likely a functional hierarchy and possibly a certain degree of redundancy exist within the smHSP family of chaperones. The differential expression of cvHSP versus HSP20 and HSP25 during muscle degeneration may reflect these variations in biological function. The abundance of larger heat shock proteins, such 
as HSP60, which is associated with mitochondrial function, and HSP70, which belongs to the main class of chaperones that bind to peptides for re-/un-folding mechanisms, were unchanged in the dystrophic diaphragm. This agrees with the idea that, in contrast to nonmuscle tissues, the musclespecific small heat shock proteins may play a more important role during fibre stress response than the HSP60 and HSP70 family of proteins. The observed decrease in GRP75, a glucose-regulated protein of the HSP70 family, and HSP90, a binding component of protein kinases and the glucocorticoid receptor complex, may be associated with impaired cell growth. This could negatively affect the repair mechanisms of damaged fibres in dystrophin-deficient skeletal muscles. On the other hand, the observed drastic increase in the very large heat shock protein HSP110 could represent a musclespecific stress response and repair mechanism in muscular dystrophy. The HSP100 family of chaperones is universally conserved and appears to exhibit an interchangeable function with HSP70 proteins.

The up-regulation of chaperones probably represents an autoprotective mechanism, whereby the stress response can be considered a reaction to the pathological increase in abnormally folded muscle proteins [51]. Interestingly, in dystrophin-deficient muscles with a decreased pathology, such as in MDX mice fed an iron-deficient diet, a reduction in HSP70 expression was observed [52]. Thus, muscle degeneration, metabolic stress and expression levels of heat shock proteins appear to correlate in muscle diseases [48, 51]. Heat shock proteins seem to play a dual role in muscular dystrophies. Firstly, they target denatured muscle proteins and then assist in their proteolytic degradation or facilitate the renaturation of malfolded peptide structures to initiate cellular repair. Secondly, during regeneration newly synthesised muscle proteins are chaperoned by various stress proteins [51]. The induction of certain small heat shock proteins may be beneficial in muscular dystrophy and the observed increase of cvHSP in the dystrophic diaphragm strengthens the concept that stress protein elements may play an important role in dystrophinopathies.

The age groups of the dystrophic animal model studied here, i.e. 9-wk- and 11-month-old MDX diaphragm fibres, covers the established period of initial muscle degeneration and the later onset fibre abnormalities in muscular dystrophy, respectively [18]. In contrast to the relatively mild symptoms seen in MDX limb muscles, the MDX diaphragm shows severe muscle wasting [17]. Following degenerationregeneration cycles, established early cellular changes that are usually observed in MDX fibres of a few weeks of age are segmental necrosis and central nucleation [18]. These histological abnormalities are associated pathobiochemically with the loss of dystrophin-associated glycoproteins (sarcoglycans, dystroglycans, sarcospan, dystrobrevins, syntrophins), $\mathrm{Ca}^{2+}$-binding proteins (calsequestrin, sarcalumenin, regucalcin) and dystrophin-linked enzymes (neuronal isoform of nitric oxide synthase) [16]. In contrast to young MDX muscles, a drastic increase in collagen deposits is present in several months old MDX fibres, which is a similarity to the severely dystrophic fibres from patients afflicted with Duchenne muscular dystrophy [12]. The immunoblot analysis of cvHSP, presented in this report, indicates that the increase in expression of this small heat shock protein intensifies with the age of the MDX mouse. There appears to be a correlation between the severity of the muscle degeneration process and the cellular stress response. With respect to developing novel therapeutic strategies to counteract muscle degeneration by targeting downstream events [53, 54], modulating the stress response might be a possibility. That this is a realistic biomedical option has recently been shown in cellbased therapy approaches. Heat shock treatment appears to enhance the survival of transplanted myoblasts [55]. Since up-regulation of heat shock proteins represents an autoprotective mechanism during biological stress, the pharmacological or cell therapeutic enhancement of this natural response may be useful for counteracting excessive muscle wasting in X-linked muscular dystrophy.

This work was supported by a principal investigator grant from Science Foundation Ireland (SFI-04/IN3/B614), a project grant from Muscular Dystrophy Ireland (MDI-2003) and a training and mobility grant from the European Commission (RTN2-2001-00337), as well as equipment grants from the Irish Health Research Board (HRB-EQ/2003/3, HRB-EQ/2004/2). The authors thank Dr. Peter Heimann (Department of Cell Biology, University of Bielefeld, Germany) for his generous help in obtaining aged MDX mice and Dr. Ica Dix (NUI Maynooth) for help with confocal microscopy. The Irish Higher Education Authority and the Deutscher Akademischer Austauschdienst supported laboratory visits of the Maynooth team in Germany.

\section{References}

[1] Cutler, P., Protein Purification Protocols, 2nd Edn., Methods in Molecular Biology, Human Press, Totowa, NJ 2004, Vol. 244.

[2] Engel, A. G., Franzini-Armstrong, C., Myology: Basic and Applied, 2nd Edn., McGraw-Hill, New York 1994.

[3] de Hoog, C. L., Mann, M., Annu. Rev. Genomics Hum. Genet. 2004, 5, 267-293.

[4] Isfort, R. J., J. Chromatogr. 2002, B771, 155-165.

[5] Yan, J. X., Harry, R. A., Wait, R., Welson, S. Y. et al., Proteomics 2001, 1, 424-434.

[6] Sanchez, J. C., Chiappe, D., Converset, V., Hoogland, C. et al., Proteomics 2001, 1, 136-163.

[7] Unlu, M., Morgan, M. E., Minden, J. S., Electrophoresis 1997, 18, 2071-2077.

[8] Tonge, R., Shaw, J., Middleton, B., Rowlinson, R. et al., Proteomics 2001, 1, 377-396.

[9] Marouga, R., David, S., Hawkins, E., Anal. Bioanal. Chem. 2005, 382, 669-678.

[10] Emery, A. E., Neuromusc. Disord. 2002, 12, 343-349. 
[11] Winder, S. J., Molecular Mechanisms of Muscular Dystrophies, Landes Bioscience, Georgetown, TX 2005.

[12] Emery, A., Muntoni, F., Duchenne Muscular Dystrophy, 3rd Edn., Oxford University Press, Oxford 2003.

[13] Ahn, A. H., Kunkel, L. M., Nat. Genet. 1993, 3, 283-291.

[14] Cohn, R. D., Campbell, K. P., Muscle Nerve 2000, 32, 14561471.

[15] Rando, T. A., Microsc. Res. Tech. 2001, 55, 223-235.

[16] Culligan, K. G., Mackey, A. J., Finn, D. M., Maguire, P. B., Ohlendieck, K., Int. J. Mol. Med. 1998, 2, 639-648.

[17] Stedman, H. H., Sweeney, H. L., Shrager, J. B., Maguire, H. C. et al., Nature 1991, 352, 536-539.

[18] Watchko, J. F., O’Day, T. L., Hoffman, E. P., J. Appl. Physiol. 2002, 93, 407-417.

[19] Okumura, N., Hashida-Okumura, A., Kita, K., Matsubae, M. et al., Proteomics 2005, 5, 2896-2906.

[20] Donoghue, P., Doran, P., Dowling, P., Ohlendieck, K., Biochim. Biophys. Acta 2005, 1752, 166-176.

[21] Kanski, J., Hong, S. J., Schoneich, C., J. Biol. Chem. 2005, 280, 24261-24266.

[22] Cieniewski-Bernard, C., Bastide, B., Lefebvre, T., Lemoine, J. et al., Mol. Cell. Proteomics 2004, 3, 577-585.

[23] Bouley, J., Meunier, B., Chambon, C., De Smet, S. et al., Proteomics 2005, 5, 490-500.

[24] Tkatchenko, A. V., Le Cam, G., Leger, J. J., Dechesne, C. A., Biochim. Biophys. Acta 2000, 1500, 17-30.

[25] Chen, Y. W., Zhao, P., Borup, R., Hoffman, E. P., J. Cell Biol. 2000, 151, 1321-1336.

[26] Bakay, M., Zhao, P., Chen, J., Hoffman, E. P., Neuromuscul. Disord. 2002, 12, S125-S141.

[27] Porter, J. D., Merriam, A. P., Leahy, P., Gong, B., Khanna, S., Hum. Mol. Genet. 2003, 12, 1813-1821.

[28] Porter, J. D., Merriam, A. P., Leahy, P., Gong, B. et al., Hum. Mol. Genet. 2004, 13, 257-269.

[29] Ge, Y., Molloy, M. P., Chamberlain, J. S., Andrews, P. C., Electrophoresis 2004, 25, 2576-2585.

[30] Ge, Y., Molloy, M. P., Chamberlain, J. S., Andrews, P. C., Proteomics 2003, 3, 1895-1903.

[31] Doran, P., Dowling, P., Lohan, J., McDonnell, K. et al., Eur. J. Biochem. 2004, 271, 3943-3952.

[32] Doran, P., Dowling, P., Donoghue, P., Buffini, M., Ohlendieck, K., Biochim. Biophys. Acta, 2006, 1764, 773-785.

[33] Sicinski, P., Geng, Y., Ryder-Cook, A. S., Barnard, E. A. et al., Science 1989, 244, 1578-1580.
[34] Towbin, H., Staehelin, T., Gordon, J., Proc. Natl. Acad. Sci. USA 1979, 76, 4350-4354.

[35] Dowling, P., Doran, P., Ohlendieck, K., Biochem. J. 2004, 379, 479-488.

[36] Jarrold, B., DeMuth, J., Greis, K., Burt, T., Wang, F., Electrophoresis 2005, 26, 2269-2278.

[37] Alderton, J. M., Steinhardt, R. A., J. Biol. Chem. 2000, 275, 9452-9460.

[38] Mallouk, N., Jacquemond, V., Allard, B., Proc. Natl. Acad. Sci. USA 2000, 97, 4950-4955.

[39] Clarke, M. S., Khakee, R., McNeil, P. L., J. Cell Sci. 1993, 106, 121-133.

[40] Attaix, D., Ventadour, S., Codran, A., Bechet, D. et al., Essays Biochem. 2005, 41, 173-186.

[41] Agbulut, O., Noirez, P., Butler-Browne, G., Jockusch, H., FEBS Lett. 2004, 562, 191-194.

[42] Morimoto, R. I., Santoro, M. G., Nat. Biotechnol. 1998, 16, 833-838.

[43] Lund, P., Molecular Chaperones in the Cell, Oxford University Press, Oxford 2001.

[44] Golenhofen, N., Der Perng, M., Quinlan, R. A., Drenckhahn, D., Histochem. Cell Biol. 2004, 122, 415-425.

[45] Krief, S., Faivre, J. F., Robert, P., le Douarin, B. et al., J. Biol. Chem. 1999, 274, 36592-36600.

[46] de Jong, W. W., Leunissen, J. A., Voorter, C. E., Mol. Biol. Evol. 1993, 10, 103-126.

[47] Nicholl, I. D., Quinlan, R. A., EMBO J. 1994, 13, 945-953.

[48] Bornman, L., Polla, B. S., Lotz, B. P., Gericke, G. S., Muscle Nerve 1995, 18, 23-31.

[49] Berchtold, M. W., Brinkmeier, H., Muntener, M., Physiol. Rev. 2000, 80, 1215-1265.

[50] Ohlendieck, K., in: Winder, S. J. (Ed.), Molecular Mechanisms of Muscular Dystrophies, Landes Bioscience, Georgetown, TX 2005, pp. 188-197.

[51] Nishimura, R. N., Sharp, F. R., Muscle Nerve 2005, 32, 693 709.

[52] Bornman, L., Rosouw, H., Gericke, G. S., Polla, B. S., Biochem. Pharmacol. 1998, 56, 751-757.

[53] Khurana, T. S., Davies, K. E., Nat. Rev. Drug. Discov. 2003, 2, 379-390.

[54] Tidball, J. G., Wehling-Henricks, M., Pediatr. Res. 2004, 56, 831-841.

[55] Bouchentouf, M., Benabdallah, B. F., Tremblay, J. P., Transplantation 2004, 77, 1349-1356. 